\title{
ON DIRECTRIX CURVES OF QUINTIC SCROLLS.
}

BY MR. CHARLES H. SISAM.

(Read before the American Mathematical Society, April 25, 1903.)

IN various papers on quintic scrolls, particular forms of the surface have been found to contain a conic. The results are all included in the following theorem :

Every unicursal quintic scroll has three coplanar generators.

Let $P_{1}, P_{2}$ be two points of the nodal curve; let $\pi_{1}, \pi_{2}$ be the planes passing through $P_{1}, P_{2}$ respectively and containing the two generators which pass through those points.

The residual curve in each plane will be a nodal cubic. These two cubics may be expressed thus :

(1) $x=a \lambda^{3}+b \lambda^{2}+c \lambda, \quad y=a^{\prime} \lambda^{3}+b^{\prime} \lambda^{2}+c^{\prime} \lambda+d^{\prime}$,

$$
z=a^{\prime \prime} \lambda^{3}+b^{\prime \prime} \lambda^{2}+c^{\prime \prime} \lambda, \quad w=0 \text {. }
$$

(2) $x=a_{1} \lambda^{3}+\cdots, \quad y=a_{1}^{\prime} \lambda^{3}+\cdots, \quad z=0, \quad w=a_{1} \lambda^{3}+\cdots$

The point $\lambda=0$ is on both curves.

The generator's join the point $\lambda$ in (1) to the point $\lambda$ in (2). If then three values $\lambda$ can be found such that the six corresponding points are coplanar, the theorem will be established.

The line

$$
w=0, \quad l x+m y+n z=0
$$

lies in the plane $\pi_{1}$ and intersects the line

$$
z=0, \quad l x+m y+n^{\prime} w=0
$$

which lies in the plane $\pi_{2}$.

The points in which the line (3) cuts the cubic (1) are defined by the values of $\lambda$ which satisfy the equation

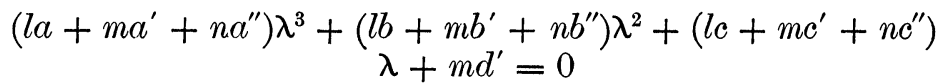

and a similar cubic defines the points in which the line (4) cuts the cubic (2). If the values of $l, m, n, n^{\prime}$ can be so chosen 
that the roots of these equations are respectively equal, then three generators intersect both the lines (3) and (4) and therefore lie in the plane determined by them.

It is no loss of generality to put $d^{\prime}=d_{1}^{\prime}$ and $n^{\prime}=1$. The conditions for the equality of the roots are now expressed by three linear equations in $l, m, n$. The solution is in general unique. The residual curve in the plane of the three generators is then a conic. There may be an infinite number of solutions. In this case the planes so determined all pass through a line which is a double directrix of the surface. Again, the scroll may be of such a form that every plane through two generators contains two other generators. The residual curve is then a rectilinear directrix. Hence :

Every unicursal quintic scroll contains either a simple conic, a double directrix line or a simple rectilinear directrix. In each case the curve is unique and constitutes the simplest directrix curve on the surface.*

If a real quintic scroll has a four-fold line and a simple rectilinear directrix, the asymptotic lines are rational quartic curves when a certain condition is satisfied. In this case, through every real point of the four-fold. line - except the pinchpoints - pass two real and two imaginary generators.

Let the equations of the scroll be

in which

$$
z \phi(x, y)+w \phi_{1}(x, y)=0 .
$$

and

$$
\phi(x, y) \equiv a x^{4}+4 b x^{3} y+6 c x^{2} y^{2}+4 d x y^{3}+e y^{4},
$$

$$
\phi_{1}(x, y) \equiv a_{1} x^{4}+\cdots ;
$$

let $x / y=\lambda, w / z=\mu$. The equation becomes

$$
\phi(\lambda)+\mu \phi_{1}(\lambda)=0 .
$$

The equation of the asymptotic lines is

$$
\frac{d \mu}{d \lambda}=k^{2} \frac{y^{2}}{z^{2}} .
$$

* Cf. Snyder, "On the quintic scroll having three double conics," BuLLETIN, vol. 9, p. 236. 
The necessary and sufficient condition that the equation of the asymptotic lines be factorable is that $d \mu / d \lambda$ be a square, or that three unequal values of $\mu$, as $\mu_{1}, \mu_{2}, \mu_{3}$, exist for each of which (5) will have a cubic factor. The values $\mu$ are the roots of the equation

$$
g_{2}=0 \text {. }
$$

Since $\mu_{1} \neq \mu_{2} \neq \mu_{3}$ and $g_{2}=0$ is a quadratic in $\mu$, hence $g_{2} \equiv 0$ and $\Delta=g_{2}^{3}-27 g_{3}^{2}=-27 g_{3}^{2}$ is negative for all real values of $\mu$ except $\mu_{1}, \mu_{2}, \mu_{3}$; hence two of the roots $\lambda$ in (5) are real and two are imaginary except at the pinch points. But the generators through the point $(0,0,1, w)$ lie in the planes $x=\lambda y, w$ $=\mu z$, hence two of the generators are real and two imaginary. Conversely, it can be shown that if $g_{2} \equiv 0$, the equation of the asymptotic lines is factorable, hence $g_{2} \equiv 0$ is the necessary and sufficient condition that the asymptotic lines are reducible.

Cornell UNIversity, March 27, 1903.

\section{JOSIAH WILLARD GIBBS, PH.D., LL.D. A SHORT SKETCH AND APPRECIATION OF HIS WORK IN PURE MATHEMATICS.}

Josiah Willaard Gibbs, Professor of Mathematical Physics in Yale University, died after a short illness, April 28, 1903. The loss to pure science in America occasioned by his death is difficult to overestimate, and it seems peculiarly fitting that some record should be made in this BULLETIN of the work in pure mathematics of so illustrious a member of the AMERICAN Mathematical Society. Moreover, his publications have appeared in a form lacking somewhat in perspicuity and detail, and are rather inaccessible.

No reference will be made in the following to the achievement of Professor Gibbs in his chosen field of Thermodynamics. A complete account of his work in Physics will be found in the American Journal of Science, September, 1903.* It is proposed here to give a brief appreciation of the ideas underlying the following papers, which constitute his published work in pure mathematics:

\footnotetext{
* Some paragraphs of the present sketch have already appeared in this article.
} 